PROCEEDINGS OF THE

AMERICAN MATHEMATICAL SOCIETY

Volume 127, Number 1, January 1999, Pages 139-143

S 0002-9939(99)05003-0

\title{
A UNIVERSAL FUNCTIONAL EQUATION
}

\author{
CARSTEN ELSNER
}

(Communicated by Hal L. Smith)

\begin{abstract}
It is shown that the S-chains solving Rubel's universal fourth-order differential equation also satisfy a third-order functional equation.
\end{abstract}

\section{IntroduCtion AND STATEMENT OF RESUlts}

In 1981, L. A. Rubel [3] published the following result concerning the approximation of continuous functions by solutions of algebraic differential equations (ADE):

There exists a nontrivial fourth-order ADE such that any real continuous function on the real axis can be uniformly approximated by the $C^{\infty}$-solutions of this $A D E$. One such specific $A D E$ is

$$
\begin{aligned}
3 y^{\prime 4} y^{\prime \prime} y^{\prime \prime \prime \prime 2}-4 y^{\prime 4} y^{\prime \prime \prime} y^{\prime \prime \prime \prime}+6 y^{\prime 3} y^{\prime \prime 2} y^{\prime \prime \prime} y^{\prime \prime \prime \prime}+24 y^{\prime 2} y^{\prime \prime 4} y^{\prime \prime \prime \prime} & \\
& -12 y^{\prime 3} y^{\prime \prime} y^{\prime \prime \prime 3}-29 y^{\prime 2} y^{\prime \prime 3} y^{\prime \prime \prime 2}+12 y^{\prime \prime 7}=0 .
\end{aligned}
$$

The basic idea in proving this theorem is to investigate the function

$$
y=A \cdot f(\alpha t+\beta)+B \quad(-1 \leq \alpha t+\beta \leq 1),
$$

where $A, B, \alpha$ and $\beta$ are real parameters, and where the function $f$ is given by

$$
f(s):=\int_{-1}^{s} e^{-1 /\left(1-u^{2}\right)} d u \quad(-1 \leq s \leq 1) .
$$

The graph of $f$ is called a primitive $S$-module. A survey on the theory of universal formulae and universal differential equations can be found in [1]. It seems to be obvious that the degree of the ADE given in (1.1) is best-possible. By using different $S$-modules constructed with

$$
\int_{-\pi / 2}^{s} e^{-1 / \cos u} d u \quad\left(-\frac{\pi}{2} \leq s \leq \frac{\pi}{2}\right),
$$

the author [2] gets a third-order ADE with coefficients depending on a certain $\varepsilon$-neighborhood and on the Lipschitz-class of the continuous functions.

Definition 1.1. Let $D$ be any set of real numbers, and let $P\left(u_{1}, \ldots, u_{m}\right)$ be a polynomial in $u_{1}, \ldots, u_{m}$ with integer coefficients, which does not vanish identically. A real function $y=y(x)$ with domain $D$ is said to be a local solution of a functional

Received by the editors May 2, 1997.

1991 Mathematics Subject Classification. Primary 34K05, 34A34.

Key words and phrases. Functional-differential equations, nonlinear ordinary differential equations.

(C)1999 American Mathematical Society 
equation of degree $n \geq 0$ (with respect to the polynomial $P$ ), if for every point $x_{0}$ from $D$ there are distinct rationals $r_{1}, \ldots, r_{k}$ and some $\varepsilon>0$, such that

$$
P\left(y\left(x+r_{1}\right), \ldots, y\left(x+r_{k}\right), y^{\prime}\left(x+r_{1}\right), \ldots, y^{\prime}\left(x+r_{k}\right), \ldots, y^{(n)}\left(x+r_{k}\right)\right)=0
$$

holds for every $x$ with $x_{0} \leq x \leq x_{0}+\varepsilon$ and $x+r_{\kappa} \in D(1 \leq \kappa \leq k)$.

Now we shall show that the functions from (1.2) are local solutions of a functional equation of degree 3 , and that the corresponding polynomial $P$ can be computed explicitly.

Theorem 1.1. Every real continuous function $F$ on the real axis can be uniformly approximated by $C^{\infty}$-functions, which are all local solutions of a functional equation of degree 3 . The corresponding polynomial $P\left(u_{1}, \ldots, u_{9}\right)$ has 206 terms, its greatest coefficient is 358318080000 , and it does not depend on $F$.

The polynomial $P$ from this theorem can be simplified in a certain sense. Put

$$
u:=\frac{y_{1}^{\prime} y_{1}^{\prime \prime \prime}}{y_{1}^{\prime \prime 2}}, \quad v:=\frac{y_{2}^{\prime} y_{2}^{\prime \prime \prime}}{y_{2}^{\prime \prime 2}}, \quad w:=\frac{y_{3}^{\prime} y_{3}^{\prime \prime \prime}}{y_{3}^{\prime \prime 2}}
$$

where

$$
y_{i}:=y\left(x+\frac{i-1}{n}\right) \quad\left(i=1,2,3 ; n \in \mathbb{Z}_{>0}\right) .
$$

Then $P$ can be written as a polynomial in $u, v$ and $w$ of degree 8 with respect to each variable.

\section{On Theorem 1.1: Existence proof of a functional Equation of DEGREE 3 AND THEORETICAL FOUNDATION OF ITS COMPUTATION}

The approximating function $Y$ of any continuous function $F$ is given by "Schains", which means a $C^{\infty}$-function that consists of S-modules (1.2) pieced together. L. A. Rubel [3] has proved that $F$ is approximated uniformly by certain S-chains $Y$ of this type. So it remains to prove the existence of a functional equation, having every S-chain as a local solution.

Let $x_{0}$ be any real number. Obviously, for some sufficiently large positive integer $n$ every real number $x$ with $x_{0} \leq x \leq x_{0}+1 / n$ belongs to the domain of some function

$$
y=A \cdot f(\alpha x+\beta)+B \quad(-1 \leq \alpha x+\beta \leq 1)
$$

from (1.2) and (1.3), which represents a certain section from the approximating function $Y$. Let $\alpha \geq 0$ first. Without loss of generality, we may assume that

$$
-1 \leq \alpha x_{0}+\beta<1
$$

hence, for sufficiently large $n$, we have

$$
\alpha x_{0}+\beta<1-\frac{3 \alpha}{n} \text {. }
$$

By $x_{0} \leq x \leq x_{0}+1 / n$, it follows that

or

$$
-1 \leq \alpha x+\beta<1-\frac{2 \alpha}{n},
$$

$$
-1+\frac{2 \alpha}{n} \leq \alpha x+\beta+\frac{2 \alpha}{n}<1
$$


For brevity, we define

$$
s_{i}:=\alpha x+\beta+\frac{\alpha(i-1)}{n} \quad(i=1,2,3)
$$

for any $x$ with $x_{0} \leq x \leq x_{0}+1 / n$, which means that $-1 \leq s_{i}<1$ for $i=1,2,3$. Thus, passing from $x$ to $x+1 / n$ and to $x+2 / n$, we do not leave the domain of the function $y$ in (2.1). If $\alpha<0$, we may assume that $1 \geq \alpha x_{0}+\beta>-1$ and proceed in the same way. From

$$
1 \geq \alpha x+\beta>-1-\frac{2 \alpha}{n}
$$

we conclude that $1 \geq s_{i}>-1(i=1,2,3)$.

First we treat the case where $y_{1}^{\prime \prime} y_{2}^{\prime \prime} y_{3}^{\prime \prime}(x) \neq 0$ with $y_{i}$ from (1.5). Differentiating $f$ with respect to $s$, we obtain from (1.3) that

$$
\begin{gathered}
f^{\prime}(s)=e^{-1 /\left(1-s^{2}\right)}, \quad f^{\prime \prime}(s)=\frac{-2 s}{\left(1-s^{2}\right)^{2}} e^{-1 /\left(1-s^{2}\right)}, \\
f^{\prime \prime \prime}(s)=\frac{6 s^{4}-2}{\left(1-s^{2}\right)^{4}} e^{-1 /\left(1-s^{2}\right)} .
\end{gathered}
$$

Note that $f^{\prime}( \pm 1)=0, f^{\prime \prime}( \pm 1)=0$ and $f^{\prime \prime \prime}( \pm 1)=0$ do exist. Differentiating the functions $y_{i}$ from (1.5) with respect to $x$, we have, using (2.1),

$$
y_{i}^{\prime}=\alpha A f^{\prime}\left(s_{i}\right), \quad y_{i}^{\prime \prime}=\alpha^{2} A f^{\prime \prime}\left(s_{i}\right), \quad y_{i}^{\prime \prime \prime}=\alpha^{3} A f^{\prime \prime \prime}\left(s_{i}\right) \quad(i=1,2,3) .
$$

To make the desired functional equation independent from special values of $A, B$, $\alpha$ and $\beta$, we have to eliminate these parameters. Thus, we get for every $i=1,2,3$ :

$$
\begin{aligned}
y_{i}^{\prime} y_{i}^{\prime \prime \prime} & =\alpha^{4} A^{2} f^{\prime}\left(s_{i}\right) f^{\prime \prime \prime}\left(s_{i}\right)=\frac{\left(6 s_{i}^{4}-2\right) \alpha^{4} A^{2}}{\left(1-s_{i}^{2}\right)^{4}} e^{-2 /\left(1-s_{i}^{2}\right)} \\
& =\frac{3 s_{i}^{4}-1}{2 s_{i}^{2}} y_{i}^{\prime \prime 2} \quad(\text { by }(2.2)) .
\end{aligned}
$$

From (1.4), we conclude for $y_{i}^{\prime \prime} \neq 0$ :

$$
u=\frac{3 s_{1}^{4}-1}{2 s_{1}^{2}}, \quad v=\frac{3 s_{2}^{4}-1}{2 s_{2}^{2}}, \quad w=\frac{3 s_{3}^{4}-1}{2 s_{3}^{2}} .
$$

In what follows, we work with $w$; there are no essential differences in using $u$ or $v$. Since $s_{3}^{2} \geq 0$, we can solve the equation for $s_{3}^{2}$ :

$$
s_{3}^{2}=\frac{w}{3}+\sqrt{\left(\frac{w}{3}\right)^{2}+\frac{1}{3}} .
$$

Let $\sigma_{3}$ denote the sign of $s_{3}$; hence

$$
s_{3}=\sigma_{3} \sqrt{\frac{w}{3}+\sqrt{\left(\frac{w}{3}\right)^{2}+\frac{1}{3}}} .
$$

Note that $\sigma_{3} \in\{-1,1\}$ depends on $x$. It is $s_{3} \neq 0$, since otherwise $f^{\prime \prime}\left(s_{3}\right)$ and $y_{3}^{\prime \prime}$ vanish by (2.2) and (2.3). But this contradicts our assumption on $y_{3}^{\prime \prime}$. We also have

$$
s_{2}=\sigma_{2} \sqrt{\frac{v}{3}+\sqrt{\left(\frac{v}{3}\right)^{2}+\frac{1}{3}}} \quad\left(\sigma_{2}=\operatorname{sign} s_{2} \in\{-1,1\}\right)
$$


and

$$
s_{1}=\sigma_{1} \sqrt{\frac{u}{3}+\sqrt{\left(\frac{u}{3}\right)^{2}+\frac{1}{3}}} \quad\left(\sigma_{1}=\operatorname{sign} s_{1} \in\{-1,1\}\right) .
$$

There is a simple relation between $s_{1}, s_{2}$ and $s_{3}$, namely

$$
2 s_{2}=2 \alpha x+2 \beta+\frac{2 \alpha}{n}=s_{1}+s_{3},
$$

or

$$
2 s_{1} s_{3}=4 s_{2}^{2}-s_{1}^{2}-s_{3}^{2} .
$$

Squaring this equation a second time, we get

$$
4 s_{1}^{2} s_{3}^{2}=\left(4 s_{2}^{2}-s_{1}^{2}-s_{3}^{2}\right)^{2} .
$$

Putting in the expressions for $s_{1}, s_{2}$ and $s_{3}$ and multiplying by 9 , we get an algebraic relation between $u, v$ and $w$ :

$$
\begin{aligned}
4 \cdot(u & \left.+\sqrt{u^{2}+3}\right)\left(w+\sqrt{w^{2}+3}\right) \\
& =\left\{4\left(v+\sqrt{v^{2}+3}\right)-u-\sqrt{u^{2}+3}-w-\sqrt{w^{2}+3}\right\}^{2} .
\end{aligned}
$$

This equation and (1.4) prove that every S-chain is a local solution of a certain functional equation of degree 3 . Note that the cases where $y_{1}^{\prime \prime} y_{2}^{\prime \prime} y_{3}^{\prime \prime}(x)=0$ do not belong to any essential exception, since one may argue with the continuity of all occurring functions. Therefore the functional equation holds for such $x$, too.

Similar to the proof of Rubel's universal differential equation, a surprising amount of cancellation takes place if we eliminate the square roots in (2.4) step by step. First we introduce some useful notations:

$$
\begin{gathered}
a:=u^{2}+3, \quad b:=v^{2}+3, \quad c:=w^{2}+3 ; \\
A:=4 v-u+w, \quad B:=-4(4 v-u-w), \quad C:=4 v+u-w, \\
D:=u w+4 u v+4 v w-u^{2}-16 v^{2}-w^{2}-27 .
\end{gathered}
$$

Hence, (2.4) takes the form

$$
A \sqrt{a}+B \sqrt{b}+C \sqrt{c}=-(4 \sqrt{a b}+\sqrt{a c}+4 \sqrt{b c}+D) .
$$

Squaring both sides, we get

$$
E \sqrt{a b}+F \sqrt{a c}=G \sqrt{b c}+H
$$

where

$$
\begin{aligned}
& E:=2 A B-8 D-8 c, \\
& F:=2 A C-2 D-32 b, \\
& G:=8 D+8 a-2 B C, \\
& H:=D^{2}+16 a b+a c+16 b c-a A^{2}-b B^{2}-c C^{2} .
\end{aligned}
$$

Squaring again, we conclude from (2.5) that

$$
4(a E F-G H)^{2} b c=\left(b c G^{2}+H^{2}-a b E^{2}-a c F^{2}\right)^{2} .
$$

The final computation is left to a computer. Putting in all the expressions for $A, B, \ldots, H$ and for $a, b, c$, we get the polynomial $P$ described in Theorem 1.1. 


\section{NOTE ADDED IN PROOF}

In the context of Theorem 1.1 one may replace Definition 1.1 by the following more convenient definition: A real function $y=y(x)$ with domain $\mathbb{R}$ is said to be a local solution of a functional equation of degree 3 , if there is a polynomial $P\left(u_{1}, \ldots, u_{9}\right)$ such that for every point $x_{1}$ from $\mathbb{R}$ and for some sufficiently small $\varepsilon>0$ depending on $x_{1}$ the identity

$$
P\left(y^{\prime}\left(x_{1}\right), y^{\prime}\left(\frac{x_{1}+x_{2}}{2}\right), y^{\prime}\left(x_{2}\right), y^{\prime \prime}\left(x_{1}\right), y^{\prime \prime}\left(\frac{x_{1}+x_{2}}{2}\right), \ldots, y^{\prime \prime \prime}\left(x_{2}\right)\right)=0
$$

holds for every real $x_{2}$ with $0 \leq x_{2}-x_{1} \leq \varepsilon$.

Instead of (1.5) put:

$$
y_{1}:=y\left(x_{1}\right), \quad y_{2}:=y\left(\frac{x_{1}+x_{2}}{2}\right), \quad y_{3}:=y\left(x_{2}\right) .
$$

Then, Theorem 1.1 remains true, and there are no essential changes within the proof.

\section{REFERENCES}

1. M. Boshernitzan, Universal formulae and universal differential equations, Annals of Mathematics 124 (1986), 273-291. MR 88a:12007

2. C. Elsner, On the approximation of continuous functions by $C^{\infty}$-solutions of third-order algebraic differential equations, Math. Nachr. 157 (1992), 235-241. MR 94i:34032

3. L. A. Rubel, A universal differential equation, Bulletin of the American Mathematical Society 4 no. 3 (1981), 345-349. MR 82e:34015

Department of Mathematics, University of Hannover, Welfengarten 1, D-30167 HanNOVER, GeRmany

E-mail address: elsner@math.uni-hannover.de 